\title{
The discrete-time Geom/G/1 queue with multiple adaptive vacations and server Setup/Closedown times*
}

\author{
Wei Sun ${ }^{1 \dagger}$, Hongke Zhang ${ }^{2}$, Naishuo Tian ${ }^{3}$ \\ 1 College of Information Science and Engineering, Yanshan University, Qinhuangdao, 066004, China \\ 2 School of Electronics and Information Engineering, Beijing Jiaotong University, Beijing, 100044, China \\ 3 College of Sciences, Yanshan University, Qinhuangdao, 066004, China
}

(Received June 8 2007, Accepted September 1 2007)

\begin{abstract}
In this paper, we discuss the discrete-time Geom/G/1 queue with multiple adaptive vacations and server setup/closedown times. We consider two types of closedown time policies which differ from each other in the completion instant of the closedown times and contrast the effects of them on the queueing system. We derive the probability generating functions (PGFs) and stochastic decomposition results of the steady-state queue length and waiting time distributions through the method of embedded Markov chain in both cases. Many discussed models related to Geom/G/1 queue are special cases of our model.
\end{abstract}

Keywords: discrete-time queues, multiple adaptive vacations, embedded Markov chain, stochastic decomposition, setup/closedown times

\section{Introduction}

Modern telecommunication systems have become more of digital systems these days than analog. It is therefore more appropriate to develop vacation models which are applicable to these systems using discrete time approach. On the other hand, the discrete-time system can be used to approximate the continuous-time system in practice. There have been fewer models developed for vacation systems in discrete time compared to literatures on continuous time models (see Doshi [2], Lee [4] and Minh [5]).

For Geom/G/1 vacation queue, most works were concentrated on the study for models of multiple vacations and single vacation. An excellent and complete study on discrete-time queueing systems with vacations has been presented by Takagi ${ }^{[8]}$. Analysis of finite and infinite buffer Geom/G/1 type queues (including batch arrivals) with different vacation policies can be found in Takagi ${ }^{[8]}$ and references therein.

In recent years, Tian and Zhang ${ }^{[9,10]}$ have considered GI/Geom/1 queue with multiple vacations. The discrete-time Geom[X]/G/1 queue under multiple vacations governed by a geometrically distributed timer has been analyzed by Fiems and Bruneel ${ }^{[3]}$. Most recently, Chang and Choi ${ }^{[1]}$ have analyzed a singleserver batch arrival bulk-service queue where customers are served in batches of random size and the server takes multiple vacations whenever queue is empty. Samanta etc ${ }^{[6]}$ have presented the discrete-time Geom $[\mathrm{X}] / \operatorname{Geom}[(\mathrm{a}, \mathrm{b})] / 1 / \mathrm{N}$ queues with single and multiple vacations, in which he considered a discrete-time single-server finite-buffer batch arrival queue in which customers were served in batches according to a general bulk-service rule. Differ from the single or multiple vacation policy, however, Zhang and Tian ${ }^{[11]}$ adopted the policy of multiple adaptive vacations. This type of limited number of vacations policy reflects the trade-off

\footnotetext{
* The authors would like to thank the support from the National Natural Science Foundation of China (No. 10671170) and the National Basic Research Program of China (No. 2007CB307100, No. 2007CB307106).

${ }^{\dagger}$ Corresponding author. Tel: +86-335-8074703

E-mail address: ysusw@yahoo.com.cn.
} 
between the benefit of working on the queue and the benefit of doing other jobs represented by the vacations. Based on this vacation policy, they got a fully new model and generalized many former results. Subsequently, Sun etc ${ }^{[7]}$ gave a steady-state analysis of the batch-arrival Geom/G/1 queue with multiple adaptive vacations.

In this paper, we consider a Geom/G/1 queueing system with multiple adaptive vacations and server setup-closedown times. Herein we will adopt a new closedown time policy which is different from the general one in the completion instant of the closedown times. As to the queueing models with closedown times, generally, a busy period begins at the moment of the closedown period completion on condition that there is at least one customer arrives. However, we mainly focus our attention on another closedown time policy, in which once there is customer arrives during the closedown times, the server immediately turns into a busy period. We will contrast the effects of the two policies on the queueing system and observe that they bring in dissimilar influences on the additional performance measures of the queueing model.

This paper is organized as follows. Description of the model and the Markov chain have been given in section 2. Then using stochastic decomposition properties, we derive the stochastic decomposition results of the model with the new closedown time policy in section 3. The PGFs for the steady-state queue length and waiting times are expressed explicitly. Contrastively, we directly give the stochastic decomposition results of the model with the general closedown time policy without proof in section 4 . Finally, we give a brief conclusion.

\section{Model description and markov chain}

Based on the classical Geom/G/1 queueing model, we introduce the multiple adaptive vacation policy and server setup-closedown times.

The arrival process of customers follows a Bernoulli process with parameter $\lambda$, that is each arrival interval $T$ is geometrically distributed with parameter $\lambda$. Let us denote by $S$ the service times of each customer, $V$ the vacation times, $U$ the setup times and $C$ the closedown times, respectively. The probability distributions and PGFs of them are listed as

$$
\begin{aligned}
& P\{S=j\}=g_{j}, j \geq 1, \quad G(z)=\sum_{j=1}^{\infty} g_{j} z^{j}, \quad P\{V=j\}=v_{j}, j \geq 1, \quad V(z)=\sum_{j=1}^{\infty} v_{j} z^{j}, \\
& P\{U=j\}=u_{j}, j \geq 1, \quad U(z)=\sum_{j=1}^{\infty} u_{j} z^{j}, \quad P\{C=j\}=c_{j}, j \geq 1, \quad C(z)=\sum_{j=1}^{\infty} c_{j} z^{j} .
\end{aligned}
$$

We assume the queueing system implements empty service and first come first service (FCFS) disciplines. In general, we also assume the arrival interval, service times, vacation times and setup/ closedown times are mutually independent.

A busy cycle begins as soon as the system becomes empty. At the moment of the last customer leaving the system, the server starts to take a closedown times with random length. Notice that we first don't adopt the general closedown time policy but the new one, that is once there is customer arrives during the closedown times, the server immediately turns into a busy period. Otherwise, the server begins to take vacations.

We consider an arrival Geom/G/1 system with vacations in which the server will take a random maximum number, denoted by $H$, of vacations provided that there are no customer arrivals during the closedown times. The probability distribution of $H$ is $P\{H=j\}=h_{j}, j \geq 1$ and the PGF $H(z)=\sum_{j=1}^{\infty} h_{j} z^{j}$. Each vacation is an i.i.d. discrete-time random variable, denoted by $V$. At each vacation completion instant, the server checks the system to see if there is any customer waiting and decides the action to take according to the state of the system. There are three cases:

- Case 1: if there is any customer waiting, the server will no longer take another vacation and begin to setup immediately.

- Case 2: if there is no customer waiting and the total number of vacations taken is still less than $H$, the server will take another vacation. 
- Case 3: if there is no customer waiting and the number of vacations taken is equal to $H$, the server will stay idle and wait for the next arrival. The server doesn't begin the setup times until there is an arrival during the idle period.

Let $J$ represent the actual number of vacations taken by the server, then

$$
J=\min \left\{H, k: V^{(k-1)}<T<V^{(k)}\right\},
$$

where $V^{(k)}$ stand for the sum of $k$ vacations $\left(V^{(0)}=0\right.$ ). Let $A_{I}$ and $A_{V}$ denote the event that the first customer arrival to an empty system occurs in an idle state and a server's vacation state, respectively. We have

$$
\begin{gathered}
P\left(A_{I}\right)=\sum_{j=1}^{\infty} h_{j} \sum_{k=j}^{\infty} P\left\{V^{(j)}=k\right\}(\bar{\lambda})^{k}=H(V(\bar{\lambda})) ; \\
P\left(A_{V}\right)=1-H(V(\bar{\lambda})) .
\end{gathered}
$$

In case 1 , the server begins his setup after the $k$ th vacation time; In case 3 , he begins his setup as soon as customer arrives. The customers arriving during the setup period as well as those arriving during the idle period are not served yet since the server is under setup. A busy period begins right after the server finishes setup period until the system becomes empty.

During busy period, define $L_{n}$ be the number of customers when the $n$th customer leaves the system and $\left\{L_{n}, n \geq 1\right\}$ be the Markov chain of the discrete-time queue length process. Then we have

$$
L_{n+1}=\left\{\begin{array}{c}
L_{n}-1+A, \quad L_{n} \geq 1, \\
Q_{b}-1+A, \quad L_{n}=0
\end{array}\right.
$$

where $A$ represents the number of customers who arrive during the internal of the $n+1$ th customer's service times and $Q_{b}$ represents the number of customers when a busy period begins. The distribution function and PGF of $A$ are as

$$
k_{j}=P\{A=j\}=\sum_{n=j}^{\infty} g_{n}\left(\begin{array}{c}
n \\
j
\end{array}\right) \lambda^{j} \bar{\lambda}^{n-j}, \quad A(z)=\sum_{j=0}^{\infty} k_{j} z^{j}=G(1-\lambda(1-z)) .
$$

Conditioning on whether there are customers arrive during the closedown times, we obtain the distribution function of $Q_{b}$ as

$$
\begin{gathered}
b_{j}=P\left\{Q_{b}=j\right\}=c_{j}+C(\bar{\lambda})\left[H(V(\bar{\lambda})) u_{j-1}+\frac{1-H(V(\bar{\lambda}))}{1-V(\bar{\lambda})} \sum_{i=1}^{j} v_{i} u_{j-i}\right], j \geq 1, \\
v_{j}=\sum_{n=j}^{\infty} v_{n}\left(\begin{array}{c}
n \\
j
\end{array}\right) \lambda^{j} \bar{\lambda}^{n-j}, \quad u_{j}=\sum_{n=j}^{\infty} u_{n}\left(\begin{array}{c}
n \\
j
\end{array}\right) \lambda^{j} \bar{\lambda}^{n-j}, c_{j}=\sum_{n=j}^{\infty} c_{n}\left(\begin{array}{c}
n \\
j
\end{array}\right) \lambda^{j} \bar{\lambda}^{n-j} .
\end{gathered}
$$

Multiplying $z^{j}$ on both sides of the distribution function and summing over $j=1,2, \cdots$, we get the PGF of $Q_{b}$ as

$$
\begin{aligned}
& Q_{b}(z)=C(1-\lambda(1-z))-C(\bar{\lambda}) \\
& +C(\bar{\lambda})\left\{H(V(\bar{\lambda})) z U(1-\lambda(1-z))+\frac{[1-H(V(\bar{\lambda}))] U(1-\lambda(1-z))(V(1-\lambda(1-z))-V(\bar{\lambda}))}{1-V(\bar{\lambda})}\right\},
\end{aligned}
$$

which yields 


$$
E\left[Q_{b}\right]=\lambda E[C]+C(\bar{\lambda})\left\{H(V(\bar{\lambda}))(\lambda E[U]+1)+\frac{1-H(V(\bar{\lambda}))}{1-V(\bar{\lambda})} \lambda[(1-V(\bar{\lambda})) E[U]+E[V]]\right\} .
$$

Therefore, the transition probability matrix of the Markov chain $\left\{L_{n}, n \geq 1\right\}$ is as

$$
P=\left[\begin{array}{ccccc}
h_{0} & h_{1} & h_{2} & h_{3} & \cdots \\
k_{0} & k_{1} & k_{2} & k_{3} & \cdots \\
& k_{0} & k_{1} & k_{2} & \cdots \\
& & k_{0} & k_{1} & \cdots \\
& & & \vdots & \vdots
\end{array}\right]
$$

where

$$
\begin{aligned}
h_{j} & =P\left\{Q_{b}-1+A=j\right\}=\sum_{i=1}^{j+1} b_{i} k_{j-i+1} \\
& =\sum_{i=1}^{j+1}\left\{c_{i}+C(\bar{\lambda})\left[H(V(\bar{\lambda})) u_{i-1}+\frac{1-H(V(\bar{\lambda}))}{1-V(\bar{\lambda})} \sum_{k=1}^{i} v_{k} u_{i-k}\right]\right\} k_{j-i+1} .
\end{aligned}
$$

Comparing the transition matrix with that of the classical Geom/G/1 queue, the difference is merely exists in the elements of the first line. Based on the structure of the transition matrix, we confirm that the system is positive recurrent if and only if $\rho=\lambda E[S]<1$.

\section{Stochastic decomposition results}

According to the analysis above, we can obviously observe that the steady-state queue length and waiting times of an arbitrary customer keep the property of stochastic decomposition. Now we will respectively give the stochastic decomposition results in Theorem 1 and Theorem 2.

Theorem 1. When $\rho=\lambda E[S]<1$, the steady-state queue length immediately after the service completions, denoted by $L_{v}$, can be decomposed into the sum of two independent random variables: $L_{v}=L_{0}+L_{d}$, where $L_{0}$ is queue length of the classical Geom/G/1 queueing system and has the PGF as

$$
L_{0}(z)=\frac{(1-\rho)(1-z) G(1-\lambda(1-z))}{G(1-\lambda(1-z))-z} ;
$$

$L_{d}$ is the additional queue length and has the PGF as

$$
\begin{aligned}
& L_{d}(z)=\frac{1}{E\left[Q_{b}\right](1-z)}\{1+C(\bar{\lambda})-C(1-\lambda(1-z))-C(\bar{\lambda}) \\
& \left.\times\left[H(V(\bar{\lambda})) z U(1-\lambda(1-z))+\frac{[1-H(V(\bar{\lambda}))] U(1-\lambda(1-z))(V(1-\lambda(1-z))-V(\bar{\lambda}))}{1-V(\bar{\lambda})}\right]\right\},
\end{aligned}
$$

where $E\left[Q_{b}\right]$ have been given in (2).

Proof. Denote $\left\{\pi_{k}, k \geq 0\right\}$ be the steady-state distribution of the queue length, which satisfies

$$
\pi_{j}=\pi_{0} h_{j}+\sum_{i=1}^{j+1} \pi_{i} k_{j-i+1}, j \geq 0 .
$$

Multiplying $z^{j}$ on both sides of the distribution function and summing over $j=0,1,2, \cdots$, we obtain

$$
L_{v}(z)=\pi_{0} \sum_{j=0}^{\infty} h_{j} z^{j}+\sum_{j=0}^{\infty} z^{j} \sum_{i=1}^{j+1} \pi_{i} k_{j-i+1}=\pi_{0} B(z)+\frac{1}{z} A(z)\left(L_{v}(z)-\pi_{0}\right),
$$


where

$$
\begin{aligned}
& B(z)=\sum_{j=0}^{\infty} z^{j} \sum_{i=1}^{j+1} c_{i} k_{j-i+1} \\
& \quad+C(\bar{\lambda})\left[H(V(\bar{\lambda})) \sum_{j=0}^{\infty} z^{j} \sum_{i=1}^{j+1} u_{i-1} k_{j-i+1}+\frac{1-H(V(\bar{\lambda}))}{1-V(\bar{\lambda})} \sum_{j=0}^{\infty} z^{j} \sum_{i=1}^{j+1} k_{j-i+1} \sum_{k=1}^{i} v_{k} u_{i-k}\right] \\
& =A(z)\left\{\frac{1}{z}(C(1-\lambda(1-z))-C(\bar{\lambda}))\right. \\
& \left.+C(\bar{\lambda})\left[H(V(\bar{\lambda})) U(1-\lambda(1-z))+\frac{1}{z} \frac{[1-H(V(\bar{\lambda}))] U(1-\lambda(1-z))(V(1-\lambda(1-z))-V(\bar{\lambda}))}{1-V(\bar{\lambda})}\right]\right\} .
\end{aligned}
$$

Substituting $A(z), B(z)$ into (5)

$$
\begin{aligned}
& L_{v}(z)=\frac{\pi_{0} G(1-\lambda(1-z))}{G(1-\lambda(1-z))-z}\{1+C(\bar{\lambda})-C(1-\lambda(1-z)) \\
& \left.-C(\bar{\lambda})\left[H(V(\bar{\lambda})) z U(1-\lambda(1-z))+\frac{[1-H(V(\bar{\lambda}))] U(1-\lambda(1-z))(V(1-\lambda(1-z))-V(\bar{\lambda}))}{1-V(\bar{\lambda})}\right]\right\} .
\end{aligned}
$$

Based on the normalization condition $L_{v}(1)=1$ and the L'Hospital rule, we get

$$
\pi_{0}=\frac{1-\rho}{E\left[Q_{b}\right]} .
$$

Substituting (6) into $L_{v}(z)$

$$
\begin{aligned}
& L_{v}(z)=\frac{(1-\rho)(1-z) G(1-\lambda(1-z))}{G(1-\lambda(1-z))-z} \frac{1}{E\left[Q_{b}\right](1-z)}\{1+C(\bar{\lambda})-C(1-\lambda(1-z))-C(\bar{\lambda}) \\
& \left.\times\left[H(V(\bar{\lambda})) z U(1-\lambda(1-z))+\frac{[1-H(V(\bar{\lambda}))] U(1-\lambda(1-z))(V(1-\lambda(1-z))-V(\bar{\lambda}))}{1-V(\bar{\lambda})}\right]\right\} .
\end{aligned}
$$

As for the above stochastic decomposition results, we give the following probability explanation. First we rewrite $L_{d}(z)$ as

$$
\begin{aligned}
& L_{d}(z)=\frac{\lambda E[C]}{E\left[Q_{b}\right]} \frac{1-C(1-\lambda(1-z))}{\lambda E[C](1-z)}+\frac{\lambda C(\bar{\lambda}) E[U]}{E\left[Q_{b}\right]} \frac{1-U(1-\lambda(1-z))}{\lambda E[U](1-z)} \\
& +\frac{\lambda C(\bar{\lambda})(1-H(V(\bar{\lambda}))) E[V]}{E\left[Q_{b}\right](1-V(\bar{\lambda}))} \frac{[1-V(1-\lambda(1-z))] U(1-\lambda(1-z))}{\lambda E[V](1-z)}+\frac{C(\bar{\lambda}) H(V(\bar{\lambda}))}{E\left[Q_{b}\right]} U(1-\lambda(1-z)) .
\end{aligned}
$$

According to the different cases of customer arrival time, we can observe the additional queue length $L_{d}$, which equals to the number of customers who arrive during the residual closedown period with probability $\lambda E[C] / E\left[Q_{b}\right]$, or the number of customers who arrive during the residual setup period with probability $\lambda C(\bar{\lambda}) E[U] / E\left[Q_{b}\right]$, or the number of customs who arrive during the residual vacation period and a whole setup period with probability $(1-H(V(\bar{\lambda}))) \lambda C(\bar{\lambda}) E[V] /\left(E\left[Q_{b}\right](1-V(\bar{\lambda}))\right)$, or the number of customers who arrive during a whole setup period with probability $C(\bar{\lambda}) H\left(V(\bar{\lambda}) / E\left[Q_{b}\right]\right.$, is diverse with different probabilities.

Now we consider the stochastic decomposition result of the steady-state waiting times of an arbitrary customer. 
Theorem 2. When $\rho=\lambda E[S]<1$, the stationary waiting times, denoted by $W_{v}$, can be decomposed into the sum of two independent random variables: $W_{v}=W_{0}+W_{d}$, where $W_{0}$ is waiting times of the classical Geom/G/1 queueing system and has the PGF as

$$
W_{0}(z)=\frac{(1-\rho)(1-z)}{(1-z)-\lambda(1-G(z))} ;
$$

$W_{d}$ is the additional delay and has the PGF as

$$
\begin{aligned}
W_{d}(z) & =\frac{1}{E\left[Q_{b}\right](1-z)}\{\lambda(1+C(\bar{\lambda}))-\lambda C(z) \\
& \left.-C(\bar{\lambda})\left[H(V(\bar{\lambda}))(z-\bar{\lambda}) U(z)+\frac{1-H(V(\bar{\lambda}))}{1-V(\bar{\lambda})} \lambda U(z)(V(z)-V(\bar{\lambda}))\right]\right\} .
\end{aligned}
$$

Proof. In the above Geom/G/1 queue with multiple adaptive vacations and server setup-closedown times, the number of customers in the system when a customer leave (except himself) is equal to the sum of customers who arrive during his waiting times and service times in case the waiting times of a customer and the latter arrival process are independent. Hence we have

$$
L_{v}(z)=W_{v}(1-\lambda(1-z)) G(1-\lambda(1-z)) .
$$

Substituting $L_{v}(z)$ given in Theorem 1 into (10)

$$
\begin{aligned}
& W_{v}(1-\lambda(1-z))=\frac{(1-\rho)(1-z)}{G(1-\lambda(1-z))-z} \frac{1}{E\left[Q_{b}\right](1-z)}\{1+C(\bar{\lambda})-C(1-\lambda(1-z)) \\
& \left.-C(\bar{\lambda})\left[H(V(\bar{\lambda})) z U(1-\lambda(1-z))+\frac{1-H(V(\bar{\lambda})) U(1-\lambda(1-z))(V(1-\lambda(1-z))-V(\bar{\lambda}))}{1-V(\bar{\lambda})}\right]\right\} .
\end{aligned}
$$

Let $1-\lambda(1-z)=z$, we get

$$
\begin{aligned}
W_{v}(z) & =\frac{(1-\rho)(1-z)}{(1-z)-\lambda(1-G(z))} \frac{1}{E\left[Q_{b}\right](1-z)}\{\lambda(1+C(\bar{\lambda}))-\lambda C(z) \\
& \left.-C(\bar{\lambda})\left[H(V(\bar{\lambda}))(z-\bar{\lambda}) U(z)+\frac{1-H(V(\bar{\lambda}))}{1-V(\bar{\lambda})} \lambda U(z)(V(z)-V(\bar{\lambda}))\right]\right\} \\
& =W_{0}(z) W_{d}(z) .
\end{aligned}
$$

Parallel to the analysis of the stationary queue length, we give the following probability explanation about the stochastic decomposition results of waiting times. First we rewrite $W_{d}(z)$ as

$$
\begin{aligned}
W_{d}(z)= & \frac{\lambda E[C]}{E\left[Q_{b}\right]} \frac{1-C(z)}{E[C](1-z)}+\frac{\lambda C(\bar{\lambda}) E[U]}{E\left[Q_{b}\right]} \frac{1-U(z)}{E[U](1-z)} \\
& +\frac{\lambda C(\bar{\lambda})(1-H(V(\bar{\lambda}))) E[V]}{E\left[Q_{b}\right](1-V(\bar{\lambda}))} \frac{[1-V(z)] U(z)}{E[V](1-z)}+\frac{C(\bar{\lambda}) H(V(\bar{\lambda}))}{E\left[Q_{b}\right]} U(z) .
\end{aligned}
$$

According to the different cases of customer arrival time, we can observe the additional delay $W_{d}$, which equals to the residual closedown period with probability $\lambda E[C] / E\left[Q_{b}\right]$, or the residual setup period with probability $\lambda C(\bar{\lambda}) E[U] / E\left[Q_{b}\right]$, or the residual vacation period and a whole setup period with probability $(1-$ $H(V(\bar{\lambda}))) \lambda C(\bar{\lambda}) E[V] /\left(E\left[Q_{b}\right](1-V(\bar{\lambda}))\right)$, or a whole setup period with probability $C(\bar{\lambda}) H\left(V(\bar{\lambda}) / E\left[Q_{b}\right]\right.$, is diverse with different probabilities. 


\section{The general closedown times}

Using the similar analytic method, now we discuss the case of adopting the general closedown times. Herein we only give the corresponding stochastic decomposition results without proof. We can contrast the results with those of the model with the new closedown time policy.

Theorem 3. When $\rho=\lambda E[S]<1$, the steady-state queue length immediately after the service completions, denoted by $L_{v}$, can be decomposed into the sum of two independent random variables: $L_{v}=L_{0}+L_{d}$, where $L_{0}$ is queue length of the classical Geom/G/1 queueing system and $L_{d}$ is the additional queue length and has the $P G F$ as

$$
\begin{aligned}
& L_{d}(z)=\frac{1-C(\bar{\lambda})}{E\left[Q_{b}\right]}+\frac{\lambda C(\bar{\lambda}) E[U]}{E\left[Q_{b}\right]} \frac{1-U(1-\lambda(1-z))}{\lambda E[U](1-z)} \\
& +\frac{\lambda C(\bar{\lambda})(1-H(V(\bar{\lambda}))) E[V]}{E\left[Q_{b}\right](1-V(\bar{\lambda}))} \frac{[1-V(1-\lambda(1-z))] U(1-\lambda(1-z))}{\lambda E[V](1-z)}+\frac{C(\bar{\lambda}) H(V(\bar{\lambda}))}{E\left[Q_{b}\right]} U(1-\lambda(1-z)),
\end{aligned}
$$

where

$$
\begin{gathered}
Q_{b}(z)=(1-C(\bar{\lambda})) z+C(\bar{\lambda})\{H(V(\bar{\lambda})) z U(1-\lambda(1-z)), \\
\left.+\frac{[1-H(V(\bar{\lambda}))] U(1-\lambda(1-z))(V(1-\lambda(1-z))-V(\bar{\lambda}))}{1-V(\bar{\lambda})}\right\}, \\
E\left[Q_{b}\right]=1-C(\bar{\lambda})+C(\bar{\lambda})\left\{H(V(\bar{\lambda}))(\lambda E[U]+1)+\frac{1-H(V(\bar{\lambda}))}{1-V(\bar{\lambda})} \lambda[(1-V(\bar{\lambda})) E[U]+E[V]]\right\} .
\end{gathered}
$$

From the above result, we can observe that the additional queue length equals to zero with probability $(1-C(\bar{\lambda})) / E\left[Q_{b}\right]$, or the number of customers who arrive during the residual setup period with probability $\lambda C(\bar{\lambda}) E[U]) / E\left[Q_{b}\right]$, or the number of customs who arrive during the residual vacation period and a whole setup period with probability $(1-H(V(\bar{\lambda}))) \lambda C(\bar{\lambda}) E[V] /\left(E\left[Q_{b}\right](1-V(\bar{\lambda}))\right)$, or the number of customers who arrive during a whole setup period with probability $C(\bar{\lambda}) H\left(V(\bar{\lambda}) / E\left[Q_{b}\right]\right.$, is diverse with different probabilities.

Theorem 4. When $\rho=\lambda E[S]<1$, the stationary waiting times, denoted by $W_{v}$, can be decomposed into the sum of two independent random variables: $W_{v}=W_{0}+W_{d}$, where $W_{0}$ is waiting times of the classical Geom/G/l queueing system and $W_{d}$ is the additional delay and has the PGF as

$$
\begin{aligned}
W_{d}(z) & =\frac{1-C(\bar{\lambda})}{E\left[Q_{b}\right]}+\frac{\lambda C(\bar{\lambda}) E[U]}{E\left[Q_{b}\right]} \frac{1-U(z)}{E[U](1-z)} \\
& +\frac{\lambda C(\bar{\lambda})(1-H(V(\bar{\lambda}))) E[V]}{E\left[Q_{b}\right](1-V(\bar{\lambda}))} \frac{[1-V(z)] U(z)}{E[V](1-z)}+\frac{C(\bar{\lambda}) H(V(\bar{\lambda}))}{E\left[Q_{b}\right]} U(z) .
\end{aligned}
$$

From the above result, we can observe that the additional delay equals to zero with probability $(1-$ $C(\bar{\lambda})) / E\left[Q_{b}\right]$, or the residual setup period with probability $\left.\lambda C(\bar{\lambda}) E[U]\right) / E\left[Q_{b}\right]$, or the residual vacation period and a whole setup period with probability $(1-H(V(\bar{\lambda}))) \lambda C(\bar{\lambda}) E[V] /\left(E\left[Q_{b}\right](1-V(\bar{\lambda}))\right)$, or a whole setup period with probability $C(\bar{\lambda}) H\left(V(\bar{\lambda}) / E\left[Q_{b}\right]\right.$, is diverse with different probabilities. 


\section{Conclusion}

In this paper we mainly give the stochastic decomposition results of the Geom/G/1 queue with multiple adaptive vacations and server setup/closedown times. We have compared the effect of the new type of closedown time policy with that of the general closedown time policy and observed that the corresponding additional performance measures of the queueing model caused by the multiple adaptive vacations and server setup/closedown times in both situations are obviously different with each other. Many of the queueing models related to Geom/G/1 queue can be regarded as the special cases of our model. We will continue to study the phase-type $(\mathrm{PH})$ closure properties of the performance measures and the busy period of the queue. Furthermore, the aspect of application problem of the model in communication network, automatic control and system engineering will also be researched in future.

\section{References}

[1] S. H. Chang, D. W. Choi. Performance analysis of a finite-buffer discrete-time queue with bulk arrival, bulk service and vacations. Computers and Operations Research, 2005, 32: 2213-2234.

[2] B. T. Doshi. Queueing systems with vacations-a survey. Queueing Systems, 1986, 1: 29-66.

[3] D. Fiems, H. Bruneel. Analysis of a discrete-time queueing system with timed vacations. Queueing Systems, 2002, 42: 243-254.

[4] S. S. Lee. Operating characteristics of batch queues with threshold and vacations. Ph.D. Dissertation, 1993. Sung Kyun Kwan University, Korea.

[5] D. L. Minh. Transient solutions for some exhaustive $\mathrm{m} / \mathrm{g} / 1$ queues with generalized independent vacations. European J. Oper. Res., 1988, 36: 197-201.

[6] S. K. Samanta, M. L. Chaudhry, U. C. Gupta. Discrete-time $g e o^{x} / g^{(a, b)} / 1 / n$ queues with single and multiple vacations. Mathematical and Computer Modelling, 2007, 45: 93-108.

[7] W. Sun, N. Tian, S. Li. Steady state analysis of the batch arrival geo/g/1 queue with multiple adaptive vacations. International Journal of Management Science and Engineering Management, 2007, 2(2): 83-97.

[8] H. Takagi. Queueing analysis. Discrete-time Systems, 1993, 3.

[9] N. Tian, Z. G. Zhang. The discrete-time gi/geo/1 queue with multiple vacations. Queueing Systems, 2002, 40: 283-294.

[10] N. Tian, Z. G. Zhang. Vacation Queueing Models: Theory and Applications. Springer-Verlag, New York, 2006.

[11] Z. G. Zhang, N. Tian. Discrete time geom/g/1 queue with multiple adaptive vacations. Queueing Systems, 2001, 38(4): 419-430. 